\title{
Design, characterization, and biological evaluation of curcumin-loaded surfactant-based systems for topical drug delivery
}

This article was published in the following Dove Press journal:

International Journal of Nanomedicine

8 September 2016

Number of times this article has been viewed

\section{Bruno Fonseca-Santos \\ Aline Martins dos Santos \\ Camila Fernanda Rodero \\ Maria Palmira Daflon \\ Gremião \\ Marlus Chorilli}

School of Pharmaceutical Sciences, UNESP - São Paulo State University, Araraquara, São Paulo Brazil
Correspondence: Maria Palmira Daflon Gremião; Marlus Chorilli

School of Pharmaceutical Sciences, UNESP - São Paulo State University, Rodovia Araraquara-Jaú km 0I, Araraquara, São Paulo I480I-902, Brazil $\mathrm{Tel}+551633016975$

+551633016998 (MC)

Email pgremiao@fcfar.unesp.br; chorilli@fcfar.unesp.br
Abstract: From previous studies, it has been found that curcumin exhibits an anti-inflammatory activity and is being used for the treatment of skin disorders; however, it is hydrophobic and has weak penetrating ability, resulting in poor drug transport through the stratum corneum. The aim of this study was to develop liquid crystalline systems for topical administration of curcumin for the treatment of inflammation. These liquid crystalline systems were developed from oleic acid, polyoxypropylene (5) polyoxyethylene (20) cetyl alcohol, and water as the surfactant, oil phase, and aqueous phase, respectively. These systems were characterized, and polarized light microscopy showed anisotropy with lamellar mesophases (Formulation 1) and hexagonal mesophases (Formulations 2 and 3), which were confirmed by the peak ratio measured using small-angle X-ray scattering. In addition, rheological tests revealed that the formulations exhibited gel-like behavior $\left(G^{\prime}>G^{\prime \prime}\right)$, as evidenced by the increased $G^{\prime}$ values that indicate structured systems. Texture profile analysis showed that hexagonal mesophases have high values of hardness, adhesiveness, and compressibility, which indicate structured systems. In vitro studies on bioadhesion revealed that the hexagonal mesophases increased the bioadhesiveness of the systems to the skin of the pig ear. An in vivo inflammation experiment showed that the curcumin-loaded hexagonal mesophase exhibited an anti-inflammatory activity as compared to the positive control (dexamethasone). The results suggest that this system has a potential to be used as a bioadhesive vehicle for the topical administration of curcumin. Therefore, it is possible to conclude that these systems can be used for the optimization of drug delivery systems to the skin.

Keywords: lamellar mesophase, hexagonal mesophase, liquid crystalline mesophase, selfassembly structures, water-surfactant-oil based-structures, curcumin, paw edema

\section{Introduction}

Liquid crystal (LC) state, also known as a mesophase, is a special state of matter that exhibits both ordered properties of solids and flow characteristics of liquids. ${ }^{1,2}$ Surfactant-based LCs contain amphiphilic surfactants that dissolve in water ${ }^{3}$ and selfassemble into various ordered mesophases. ${ }^{4}$ These mesophases are lamellar, hexagonal, or cubic ${ }^{5}$ in shape and have attracted attention in the pharmaceutical industry as they have distinctive structures and physicochemical properties, ${ }^{4}$ such as viscosity, bioadhesion proprieties, the ability to control the release of drugs, and loading of hydrophilic or lipophilic drugs. ${ }^{6-10}$ LCs have been utilized as semisolid vehicles for topical administration of drugs. ${ }^{11-16}$

Curcumin has a long history of use in Ayurvedic medicine treatment for inflammatory conditions ${ }^{17,18}$ as it has an ability to inhibit nuclear factor $\kappa \mathrm{B} .{ }^{19}$ It has been used in the 
treatment of various skin diseases and conditions, ${ }^{20}$ especially wounds, ${ }^{21}$ psoriasis, ${ }^{22,23}$ radiation-induced dermatitis, ${ }^{24}$ allergic dermatitis, ${ }^{25}$ and skin cancer. ${ }^{26-28}$ Although it exhibits favorable biological activities on the skin, some properties of curcumin limit its potential as a therapeutic agent, such as poor water solubility and low bioavailability, ${ }^{29}$ resulting in limited skin penetration ${ }^{30}$ and poor transport through the stratum corneum. ${ }^{31}$

Bioadhesion includes adhesion to the skin, and it can be described as a formation of a mechanical joint between the surfaces of adherent (skin) and the adhesive dosage forms. Various theories have attempted to explain the bioadhesion process: there is a bonding force as a result of physicochemical interaction between skin dosage forms; viscoelastic properties can interact due to elastic-viscous behavior of these materials; or the diffusion or interpenetration can explain these adhesion processes because the molecules of dosage forms interact with the skin surface at the molecular level. ${ }^{32}$ The adhesion process is complex and can be governed by synergic/combinatory effects described by the above theories.

Numerous nanotechnology-based drug delivery systems, have been developed to enhance the solubility of drugs, such as LCs. ${ }^{33}$ The oil phase present in LCs allows for dissolution of lipophilic drugs. Nevertheless, several studies have shown that lamellar-, hexagonal-, or cubic-mesophase-based formulations are capable of penetrating the skin and act as good candidates for topical drug delivery systems. ${ }^{5,15,16,34-37}$

\section{Experimental data Materials}

Polyoxypropylene (5) polyoxyethylene (20) cetyl alcohol was purchased from Volp Indústria Comércio (Osasco, São Paulo, Brazil), and oleic acid was purchased from Synth (Diadema, São Paulo, Brazil). Lambda carrageenan and curcumin were acquired from Sigma-Aldrich (St Louis, MO, USA). Commercial dermatological cream containing dexamethasone acetate $(1 \mathrm{mg} / \mathrm{g})$ was purchased from local pharmacy (Araraquara, São Paulo, Brazil). Porcine ears were acquired from a local slaughterhouse (Tupã, São Paulo, Brazil).

\section{Ternary phase diagram}

This diagram was constructed by combining PPG-5CETETH-20, oleic acid, and water as described by Carvalho et al amongst others. ${ }^{38,39}$ The percentages of these three components range from $10 \%-80 \%(\mathrm{w} / \mathrm{w})$ and were calculated to obtain the points that defined the boundaries between the regions of the ternary phase diagram. After 24 hours, the formulations were visually classified by using phase separation, opacity, viscosity systems, and by translucency. Three formulations were selected for further physicochemical characterization (Table 1). In these formulations, curcumin was loaded at a concentration of $5 \mathrm{mg} / \mathrm{g}$.

\section{Polarized light microscopy}

Samples for polarized light microscopy (PLM) were prepared by placing a drop of each formulation between a coverslip and a glass slide, and in order to analyze various visual fields of each sample, these samples were examined under a polarized light using a polarized light microscope (Axioskop ${ }^{\circledR}$, Zeiss, Germany) at room temperature. The isotropic and anisotropic behaviors of the samples were examined, and photomicrographs were obtained at $\times 40$ magnification.

\section{Small-angle X-ray scattering}

Data were collected using the Synchrotron small-angle $\mathrm{X}$-ray scattering (SAXS) beamline at the National Laboratory of Synchrotron Light (LNLS; Campinas, Brazil). The beamline was equipped with a monochromator $(\lambda=1.488 \AA)$, a vertical detector located at $\sim 1.5 \mathrm{~m}$ from the sample, and a multichannel analyzer, which recorded the intensity of the scattering pattern $(I)$ and the scattering vector modulus $(q)$. The intensity values of the mica and air were subtracted from the total scattered intensity (I). Each spectrum was recorded for $\sim 45$ seconds, and the scattering vector modulus, $q$, was calculated at $\sim 0.1-5 \mathrm{~nm}^{-1}$. The analyses were performed at $25^{\circ} \mathrm{C} \pm 1^{\circ} \mathrm{C}$.

From the SAXS curves, the position of the first peak $\left(q_{1}\right)$ was used to assess the lattice parameters of the lamellar and hexagonal phases and the distance between planes $(d)$ and hexagon edge $(a)$ according to Equations 1 and 2, respectively:

$$
\begin{gathered}
d=\frac{2 \pi}{q_{1}} \\
d=\frac{2 \pi}{q_{1}}=\frac{\sqrt{3}}{2} a
\end{gathered}
$$

Table I Composition (\%) of water, the oil phase (oleic acid), and surfactant (PPG-5-CETETH-20) in the formulations

\begin{tabular}{llll}
\hline Formulations & \multicolumn{3}{l}{ Components (\%) } \\
\cline { 2 - 4 } & Water & Oleic acid & PPG-5-CETETH-20 \\
\hline FI & 30 & 30 & 40 \\
F2 & 40 & 20 & 40 \\
F3 & 50 & 10 & 40 \\
\hline
\end{tabular}

Abbreviation: PPG-5-CETETH-20, polyoxypropylene (5) polyoxyethylene (20) cetyl alcohol. 


\section{Rheological oscillatory analysis}

Rheological oscillatory analysis was performed using a rheometer (AR 2000EX; TA Instruments, New Castle, USA) of parallel plate-plate and cone-plate geometry, according to the consistency of each formulation. Oscillatory analysis of each sample was carried out after the linear viscoelastic region in which stress is directly proportional to strain and the storage modulus remains constant was identified. Frequency sweep analysis was carried out at the frequency range of $0.1-10 \mathrm{~Hz}$ at a constant stress of $1 \mathrm{~Pa}$. All measurements were taken at $25^{\circ} \mathrm{C} \pm 0.25^{\circ} \mathrm{C}$.

\section{Texture profile analysis}

Texture profile analysis (TPA) was carried out by using a TA-XTplus texture analyzer (Stable Micro Systems, Surrey, UK) as described by Calixto et al. ${ }^{40}$ The samples were weighed $(7 \mathrm{~g}$ ), placed in $50 \mathrm{~mL}$ centrifuge tubes (Falcon; BD Biosciences $^{\circledR}$, Franklin Lakes, NJ, USA), and centrifuged (Sorval TC 6 centrifuge; Du Pont, Newtown, CT, USA) to remove the air bubbles present in the samples. Then, the samples were allowed to stand for 24 hours until analysis. Thereafter, each sample was placed below the $10 \mathrm{~mm}$ analytical probe, which was lowered at a constant speed of $1 \mathrm{~mm} \cdot \mathrm{s}^{-1}$ until it reached the sample. Contact was detected by a triggering force of $2 \mathrm{mN}$, and then the probe continued down to $10 \mathrm{~mm}$ depth in the sample. Then, the probe returned to the surface $\left(0.5 \mathrm{~mm} \cdot \mathrm{s}^{-1}\right)$, and after 5 seconds, a second compression was initiated. The test results were used to plot a force-time curve, from which the mechanical parameters were calculated such as hardness, compressibility, adhesiveness, and cohesion. This process was repeated seven times at $25^{\circ} \mathrm{C} \pm 0.5^{\circ} \mathrm{C}$.

\section{Bioadhesion measurement}

The bioadhesive force between the skin of the pig ear and the samples was assessed using the TA-XTplus texture analyzer as described by Cintra et al. ${ }^{41}$

Fresh ears of healthy 6-month-old pigs were obtained from a local slaughterhouse and were prepared for the analysis as described by Dick and Scott. ${ }^{42}$ The ears were cleaned with purified water $\left(25^{\circ} \mathrm{C} \pm 0.5^{\circ} \mathrm{C}\right)$, and injured ears were not included for the analysis. The undamaged skin was removed from the cartilage with a scalpel, and a $400 \mu \mathrm{m}$-thick layer (stratum corneum and epidermis) was separated from the adipose tissue with a dermatome (Nouvag TCM 300; Goldach, USA). The prepared skin samples were frozen at $-20^{\circ} \mathrm{C}$ and were stored no longer than 4 weeks.

Each skin sample was thawed in a physiological saline solution made up of $0.9 \%(\mathrm{w} / \mathrm{v}) \mathrm{NaCl}$ at $25^{\circ} \mathrm{C} \pm 0.5^{\circ} \mathrm{C}$ for
30 minutes. Then, the hairs were cut with scissors and attached to the end of a cylindrical probe (diameter, $10 \mathrm{~mm}$ ) with a rubber ring. A $50 \mathrm{~mL}$ centrifuge tube containing the samples was placed below the probe and immersed in a water bath at $32^{\circ} \mathrm{C} \pm 0.5^{\circ} \mathrm{C}$. The probe was lowered at a constant speed of $1 \mathrm{~mm} \cdot \mathrm{s}^{-1}$ until the skin and sample were made to be in contact with each other, as detected by a triggering force of $2 \mathrm{mN}$. The skin and sample were kept in contact for 60 seconds, and no external force was applied during this time interval. After 60 seconds, the skin was drawn upward $\left(0.5 \mathrm{~mm} \cdot \mathrm{s}^{-1}\right)$ until the contact between the surfaces was broken. During this experiment, a force-time curve was plotted, and the work of adhesion and peak adhesion were calculated from this plot. The process was repeated five times at $32^{\circ} \mathrm{C} \pm 0.5^{\circ} \mathrm{C}$

\section{Evaluation of anti-inflammatory effects in vivo}

The in vivo evaluation was performed on male Swiss mice (each weighed 25-35 g). The mice were kept in a temperature-controlled environment $\left(22^{\circ} \mathrm{C}\right)$ in a $12 \mathrm{~h}$ light/ dark cycle and were provided with free access to food and water, except during the experiments. The animal procedures were performed in accordance with the protocol approved by the Animal Ethics Committee of the School of Pharmaceutical Sciences - UNESP (approval number, 67/2015).

The mice were subdivided into six groups with five per group: Group I mice were not treated (negative control); Group II received topical dexamethasone (positive control); Group III received Formulation 1 (F1) without curcumin; Group IV received F1 with curcumin; Group V received F2 without curcumin; and Group VI received F2 with curcumin.

Paw edema was induced by intraplantar injection of $100 \mu \mathrm{L}$ of $1 \%(\mathrm{w} / \mathrm{v}) \lambda$-carrageenan into the paw of the mice. After 30 minutes, dexamethasone or LC formulations (100 mg) were applied to the paw. Then, 5 hours after the administration of carrageenan, the thickness of the paws were measured (in $\mathrm{mm}$ ) by using a digital micrometer. The means and standard deviation of thickness were calculated for each group. One-way analysis of variance (ANOVA) was perfomed, followed by post hoc Dunnett's test was used to detect differences between the mean of treated animals and control group were considered significant at $P<0.05$.

\section{Results and discussion \\ Ternary phase diagram}

The formulations were visually classified by using the following: phase separation that is referred to as the segregation of 
components that results in the formation of two phases; liquid translucent systems that are liquid systems that have flow properties similar to water; a low-viscosity system that tends to be translucent and has a relatively low viscosity, as indicated by the fluidity in inverted vials; a high-viscosity system that tends to adhere to vials even after inversion; opaque and milky systems that were characterized as emulsions.

The ternary phase diagram in Figure 1 shows that it was possible to obtain a liquid optically transparent system in the regions where the surfactant concentration, oil phase, and water concentration were $>30 \%, 0 \%-70 \%$, and $0 \%-30 \%$, respectively. Transparent semisolid systems were formed when the concentrations of the surfactant, oil phase, and aqueous phase were $35 \%-60 \%, 0 \%-60 \%$, and $35 \%-90 \%$, respectively. We found that an increase in the concentration of water in this region yielded more viscous gels (highviscosity system), as a result of solvation of the polar head of the surfactant, forming more organized structures such as hexagonal mesophases. ${ }^{43-45}$

Emulsion systems were obtained in the regions with a low surfactant concentration $(0 \%-30 \%)$ and a high aqueous phase concentration $(80 \%-100 \%)$. Phase separation took place when the surfactant concentration increased in the emulsion regions $(>70 \%)$ and at aqueous concentrations $>75 \%$, indicating that dilution with water resulted in poor stability.

The most popular approach to rationalization of different structures found in the lyotropic LCs is based on the critical

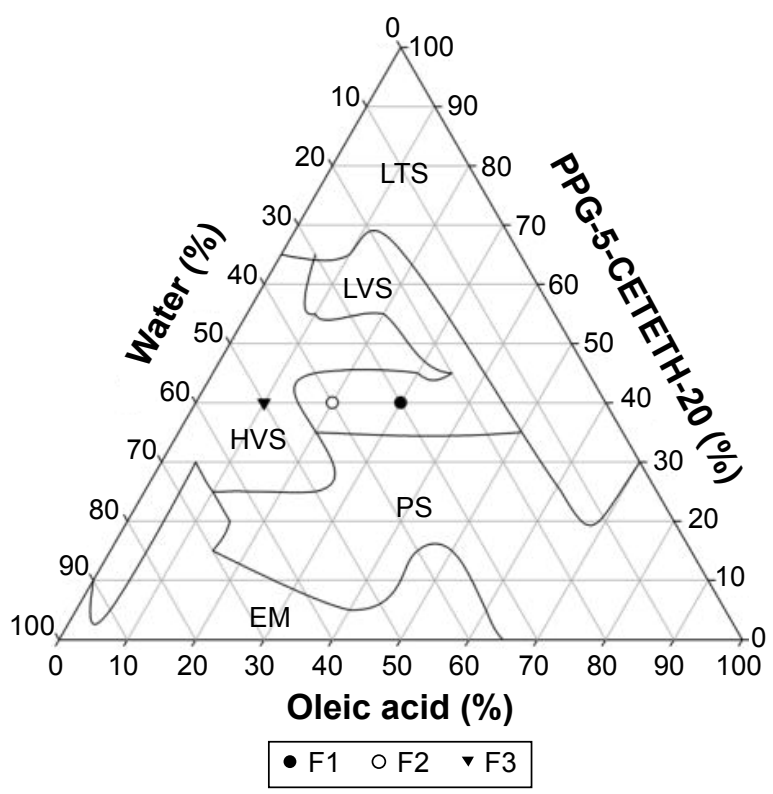

Figure I A ternary phase diagram of PPG-5-CETETH-20 (surfactant), oleic acid (oil phase), and water.

Abbreviations: EM, emulsion; HVS, high-viscosity system; LTS, liquid translucent system; LVS, low-viscosity system; PPG-5-CETETH-20, polyoxypropylene (5) polyoxyethylene (20) cetyl alcohol; PS, phase separation. packing parameter (CPP), which is equal to $v_{s} / a_{0} l \cdot{ }^{46,47} \mathrm{CPP}$ is a geometric value that constitutes the ratio of the volume of the hydrophobic tail $\left(v_{s}\right)$ to the product of volume of the polar head area $\left(a_{0}\right)$ and length of the hydrophobic tail $(l)$. A change in CPP values can roughly predict the order of surfactant transition associated with the change in the curvature of the water or oil interface. ${ }^{44}$ Increasing the number of water molecules increases the CPP values by an increase in the volume of the lipophilic moiety and a reduction in the chain length and the head-group area. ${ }^{48}$ Furthermore, this change leads to an increase in the curvature and therefore induces the formation of cubic and hexagonal mesophases. ${ }^{44,48}$

The approach of CPP is limited to a qualitative interpretation of phase diagrams and cannot assess the structural complexity of the mesophases or the supramolecular mechanisms that regulate its structural organizational behavior. ${ }^{49}$

\section{PLM and SAXS}

F1, F2, and F3 were selected for characterization as shown in the phase diagram in Figure 2; Table 2 shows their composition. The surfactant concentration was fixed at $40 \%$, whereas the proportions of water and oil varied. Figure 2 shows the PLM images.

PLM analysis of F1 showed Malta crosses, and this formulation was characterized as anisotropic, indicating lamellar mesophases. ${ }^{50}$ The lamellar phase consists of bilayers that are separated by the layers of surfactants and solvents, forming a one- or two-dimensional network. ${ }^{51}$ In contrast, F2 and F3 were characterized as hexagonal mesophases, evidenced by stretching in the photomicrographs. ${ }^{50}$ The hexagonal phase

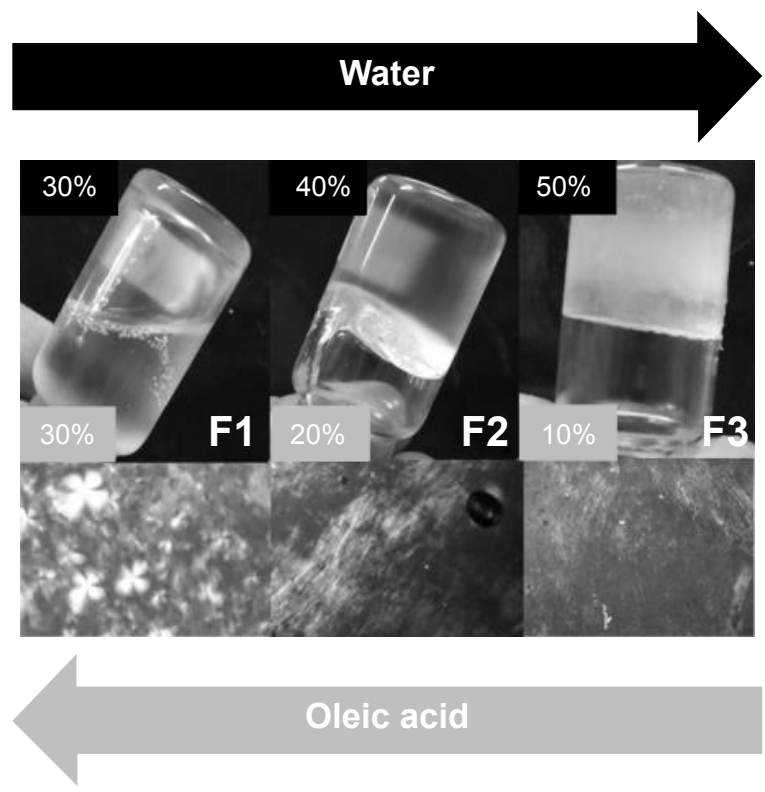

Figure 2 Macroscopic features and polarized light microscopy of the formulations (FI, F2, and F3). 
Table 2 Values of $q_{\max }\left(1 / \mathrm{nm}^{-1}\right)$, ratio of distances, and the interplanar distances $(d, a)$ of the formulations

\begin{tabular}{lllllllll}
\hline Formulations & $\mathbf{q}$ & $\boldsymbol{q}$ & $\boldsymbol{q}$ & $\boldsymbol{d}_{2} / \boldsymbol{d}_{\mathbf{1}}$ & $\boldsymbol{d}_{\mathbf{3}} / \boldsymbol{d}_{1}$ & Mesophase & $\boldsymbol{d}(\mathbf{n m})$ & $\boldsymbol{a}(\mathbf{n m})$ \\
\hline FI & 0.74 & 1.49 & - & 2 & - & Lamellar & 8.49 & - \\
F2 & 0.80 & 1.40 & 2.34 & 1.73 & 3 & Hexagonal & 7.85 & 9.06 \\
F3 & 0.78 & 1.41 & 2.34 & 1.73 & 3 & Hexagonal & 8.05 & 9.30 \\
\hline
\end{tabular}

consists of long parallel cylindrical aggregates arranged in two- or three-dimensional structures. ${ }^{52}$

SAXS was carried out in order to confirm the arrangement of the liquid crystalline formulations obtained from PLM. This method can detect the scattering of X-rays related to the interplanar distances with nanometric dimensions; thus, it can characterize systems such as droplets, micelles, and crystalline structures. ${ }^{44}$ SAXS has been widely used to elucidate the structures of liquid crystalline systems, providing information such as the size, shape, quantity, and arrangement of object spreaders in a sample. The SAXS curves for liquid crystalline systems provide the peaks and the number and ratio of the correlation distances, which can determine the type of arrangement that forms the matrix. ${ }^{53}$

Figure 3 shows the intensity of the scattering patterns $(I)$ and scattering vector modulus $\left(q ; 1 / \mathrm{nm}^{-1}\right)$. F1 yielded broad peaks of a liquid crystalline phase, indicating the presence of a less ordered phase, probably a micellar solution, in admixture with an ordered liquid crystalline phase. The ratio of the correlation distances between these samples was 1:2, suggesting the presence of lamellar mesophases. For F2 and $\mathrm{F} 3$, the ratio of correlation distances between these samples was 1:1.73:3. In lamellar phases and hexagonal phases, the $q$ values of the Bragg peaks are in the ratios 1:2:3:4:5 and 1:1.73:2:2.64:3:3.46, respectively. ${ }^{44,49,54}$

The microstructure lattice parameters are represented by the distance between planes ( $d$, lamellar structures) and the

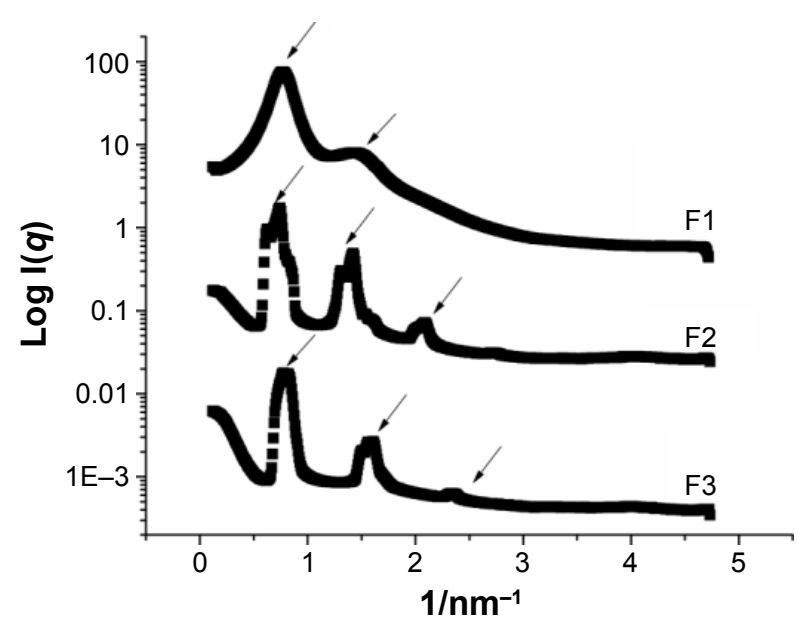

Figure 3 Small-angle X-ray scattering patterns of samples FI, F2, and F3. hexagon edge ( $a$ ) that is related to the distance between the planes passing through the two rows of adjacent cylinders ( $d$, hexagonal structure) ${ }^{55}$ The distances between planes $(d)$ were $8.49,7.85$, and $8.05 \mathrm{~nm}$ for F1, F2, and F3, respectively, and the hexagon edges (a) for F2 and F3 were 9.06 and $9.30 \mathrm{~nm}$, respectively.

\section{Rheological oscillatory analysis}

The oscillatory frequency sweep was conducted with F1, $\mathrm{F} 2$, and F3 in the absence or presence of curcumin. The storage modulus $\mathrm{G}^{\prime}$ and the loss modulus $\mathrm{G}^{\prime \prime}$ were plotted against the frequency, and Figure 4 presents the representative rheograms.

The storage modulus is a measure of energy stored and retrieved by the deformation cycle and reflects the solid characteristics of a viscoelastic material. ${ }^{56} \mathrm{~A}$ high storage modulus indicates a predominantly elastic and highly structured sample. The loss modulus is a measure of energy dissipated per cycle and reflects liquid-like characteristics. Higher values of the loss modulus mean that a sample is predominantly viscous. ${ }^{57}$

F1 was found to be less viscous than elastic $\left(G^{\prime}>G^{\prime \prime}\right)$, and the poor organization and interaction between the system components can explain the high values of loss moduli. F2 and F3 exhibited a gel-like behavior, with a higher $\mathrm{G}^{\prime}$ than $\mathrm{G}^{\prime \prime}$, both independent of frequency. The increase in the elastic moduli reflected the higher organization of F2 and F3

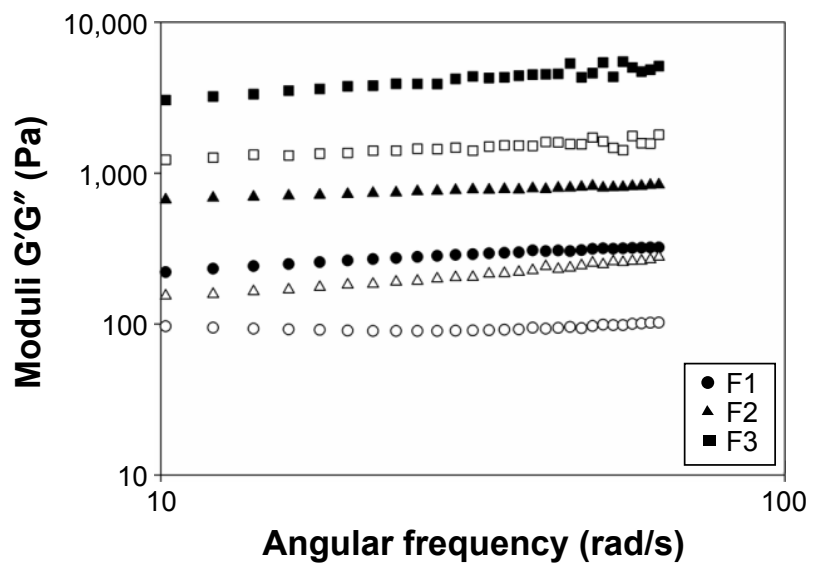

Figure 4 Storage and loss moduli vs frequency obtained by an oscillatory frequency sweep of formulations $\mathrm{FI}, \mathrm{F} 2$, and $\mathrm{F} 3$ at $25^{\circ} \mathrm{C}$.

Note: Filled symbols represent $G^{\prime}$ modulus, and open symbols represent $G^{\prime \prime}$ modulus. 
than that of F1. All samples loaded with curcumin showed the rheological profile similar to those without curcumin, indicating that this drug did not interfere with the system. The viscous rheological behavior $\left(G^{\prime \prime}>G^{\prime}\right)$ of microemulsions or liquid crystalline lamellar systems and the gel-like behavior $\left(\mathrm{G}^{\prime}>\mathrm{G}^{\prime \prime}\right)$ of hexagonal and cubic mesophases have been reported elsewhere. . $^{13,35,38,43,58,59}$

\section{TPA}

TPA is a penetrometric method that has been extensively used for mechanical characterization of food materials. ${ }^{60}$ In addition, it has emerged as a useful method in the field of pharmaceutical characterization. ${ }^{61,62}$

The parameters that can be derived from TPA include hardness (a force required to attain a required deformation), adhesiveness (a quantity that simulates the work required to overcome the attractive forces between the surface of the sample and the surface of the probe with which the sample comes into contact with the surface), compressibility (the force per unit of time required to deform the product during the first compression cycle of the probe), and cohesiveness (the force required to overcome the internal bonds of the material). ${ }^{63}$

Table 3 shows the TPA parameters of F2 and F3 (with and without curcumin). It was not possible to perform this test on F1 samples as they exhibited a liquid behavior. The TPA values of the samples differed between these two formulations. Samples with a greater aqueous phase (F3) yielded twofold higher values of hardness, compressibility, and adhesion than those with F2. The addition of the drug did not affect the mechanical behavior of the samples.

It has been reported that both the hardness and compressibility of polysaccharide gels increase when the degree of cross-linking increases. ${ }^{64}$ Some studies have shown that there is a relation between hardness and the concentration of polymers of polyacrylic acid hydrogels (eg, carbomer homopolymer type A [C971] and type B [C974] and polycarbophil)..$^{40,58}$

Few studies have elucidated the mechanical properties of liquid crystalline systems with structured mesophases.
The TPA data are in correlation with those of the rheological analysis, and in these data, the elastic modulus of the hexagonal phase was the highest. Furthermore, the results were consistent with the previous findings that hexagonal phases have high hardness and compressibility parameters. ${ }^{13,65}$

Compressibility reflects the ease of taking the prepared formulation from the container and the ease of spreading on the application site ${ }^{66}$ and adhesiveness is the work required to overcome the attractive forces between the surface of the sample and the surface of the probe. ${ }^{64}$ Formulations for cutaneous application should have both good hardness and adhesiveness. ${ }^{67}$ On the other hand, formulations with strong adhesiveness and cohesiveness ensure prolonged adhesion to the surface site and a complete structural recovery of the formulation after application. ${ }^{68}$

\section{Bioadhesion measurements}

Bioadhesion refers to the ability of synthetic, biological, and hydrocolloidal macromolecules to adhere to biological tissues. $^{32}$ The advantages of using bioadhesive systems as drug carriers include prolongation of drug residence time at the absorption site, intensified contact with the epithelial barrier, decreased frequency of drug application, and improved patient compliance with therapy. ${ }^{69}$

Table 4 shows the peak and area of bioadhesion of the formulations tested. It is shown that the lamellar system (F1) has a lower peak and work of adhesion than the hexagonal systems do (F2 and F3). This is possibly due to the type of mesophase formed because lamellar mesophases are less rigid than hexagonal mesophases that have a complex and rigid network. The peak and area of adhesion were similar to those reported for hydrogels when applied to the skin. ${ }^{58}$

Several theories have been proposed to explain bioadhesion and mucoadhesion of the formulations. ${ }^{70-78}$ Viscosity plays an important role in mucoadhesion, wherein increased viscosity of a pharmaceutical system gradually interacts with the substrate surface. ${ }^{69}$ Some hydrogels exhibit gellike behavior in situ only after exposure to an external stimulus. These are the so-called environmentally sensitive polymers and are called as thermosensitive polymers, for

Table 3 Mechanical properties (hardness, compressibility, adhesiveness, and cohesiveness) of the liquid crystal (F2 and F3) formulations with or without curcumin

\begin{tabular}{lllll}
\hline Formulations & Hardness $\mathbf{( N )}$ & Compressibility $\mathbf{( N} \cdot \mathbf{s})$ & Adhesiveness $(\mathbf{N} \cdot \mathbf{s})$ & Cohesiveness $(\mathbf{N})$ \\
\hline F2 & $0.048 \pm 0.003$ & $0.303 \pm 0.076$ & $0.329 \pm 0.025$ & $0.632 \pm 0.064$ \\
F2C & $0.054 \pm 0.008$ & $0.457 \pm 0.08 \mathrm{I}$ & $0.339 \pm 0.02 \mathrm{I}$ & $0.65 \mathrm{I} \pm 0.103$ \\
F3 & $0.163 \pm 0.008$ & $1.099 \pm 0.036$ & $1.259 \pm 0.17 \mathrm{I}$ & $0.695 \pm 0.037$ \\
F3C & $0.159 \pm 0.009$ & $1.259 \pm 0.17 \mathrm{I}$ & $1.399 \pm 0.122$ & $0.722 \pm 0.050$ \\
\hline
\end{tabular}

Note: Values are expressed as mean \pm standard deviation of seven replicates. 
Table 4 Parameters of the in vitro bioadhesion test of liquid crystals formulations with or without curcumin

\begin{tabular}{lll}
\hline Formulations & Peak of adhesion $(\mathbf{N})$ & Area of adhesion $(\mathbf{N} \cdot \mathbf{s})$ \\
\hline FI & $0.007 \pm 0.002$ & $0.068 \pm 0.011$ \\
FIC & $0.005 \pm 0.005$ & $0.060 \pm 0.014$ \\
F2 & $0.059 \pm 0.021$ & $0.098 \pm 0.010$ \\
F2C & $0.069 \pm 0.041$ & $0.095 \pm 0.012$ \\
F3 & $0.1078 \pm 0.026$ & $0.245 \pm 0.049$ \\
F3C & $0.1101 \pm 0.020$ & $0.220 \pm 0.056$ \\
\hline
\end{tabular}

Notes: Data were collected at $32^{\circ} \mathrm{C} \pm 0.5^{\circ} \mathrm{C}$. The values are represented as mean \pm standard deviation of five replicates.

example, poloxamers. ${ }^{79-81}$ These stimuli exist in organisms, highlighting the possible suitability of such polymers for the development of controlled release and bioadhesive systems as they have stimuli-induced viscosity. ${ }^{82-85}$ Mucoadhesion of thermosensitive gels and LCs can be explained by their rheological properties. ${ }^{39,41,86-91}$ Moreover, the mucoadhesive force of the liquid crystalline phases is determined by their ability to take up water from the environment. ${ }^{87}$ Many theories have attempted to explain the biomucoadhesion; however, for the systems developed, the elastic behavior $\left(G^{\prime}>G^{\prime \prime}\right)$ has an influence on the bioadhesive process. Materials with elastic properties are shown to be optimal for good mucoadhesion or bioadhesion. ${ }^{13,40,43,58,92}$

\section{Evaluation of anti-inflammatory effects in vivo}

These effects were evaluated as follows, and the following groups were analyzed: untreated (negative control), dexamethasone (positive control), curcumin-loaded sunflower, curcumin-loaded LC formulations (F1C and F2C), and LC vehicles (F1 and F2). Figure 5 shows the in vivo results of

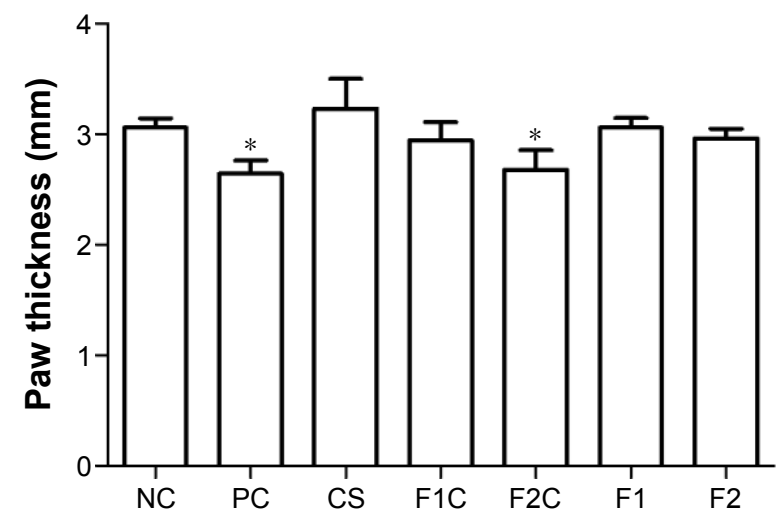

Figure 5 Anti-inflammatory activity of the LCs with curcumin at $5 \mathrm{mg} \mathrm{g}^{-1}$ (FIC and $\mathrm{F} 2 \mathrm{C}$ ), LC vehicles (FI and F2), PC (cream containing dexamethasone at $\mathrm{I} \mathrm{mg} \mathrm{g}^{-1}$ ), and NC (untreated).

Notes: The data are represented mean \pm standard deviation of results on five mice. The statistical significance of paw thickness was analyzed using variance analysis by Dunnett's multiple comparison test; $* P<0.05$.

Abbreviations: LC, liquid crystal; NC, negative control; PC, positive control. the anti-inflammatory activity of curcumin incorporated in the systems. Compared with dexamethasone (positive control), the inhibition was similar to that of the curcumin-loaded hexagonal mesophase (F2C). Curcumin-loaded hexagonal mesophase exhibited an ability to reduce paw edema possibly owing to the high bioadhesive ability, which increased the contact time with the skin surface, thus promoting the absorption of curcumin. The LC vehicles (F1 and F2) did not influence anti-inflammatory activity, according to edema inhibition in the mouse paw. Curcumin-loaded LC formulation (F1C) and curcumin-loaded sunflower did not inhibit edema; this result implies a poor bioadhesive ability of these systems.

\section{Conclusion and future perspectives}

The mixture of polyoxypropylene (5) polyoxyethylene (20) cetyl alcohol, oleic acid, and purified water exhibited the ability to form liquid crystalline lamellar phase systems or hexagonal phase systems. In addition, the hexagonal phases exhibited good bioadhesive properties on the skin and an antiinflammatory activity, and the latter effect was comparable to that of dexamethasone-containing commercial cream (positive control). Furthermore, curcumin in an oil solution or lamellar mesophases did not exhibit the anti-inflammatory activity as it has poor bioadhesive ability or probably poor ability to interact with the skin surface interface. Thus, it was concluded that hexagonal mesophase systems are promising drug delivery platforms and can be used as a vehicle for topical administration of curcumin, as demonstrated by the beneficial biological activity in vivo. Thus, this work showed the possibility of incorporating curcumin in a new vehicle for topical application for the treatments of skin diseases using this curcumin-loaded vehicle.

\section{Acknowledgments}

The authors are grateful to Coordination for the Improvement of Higher Level Personnel (CAPES), National Council for Scientifc and Technological Development (CNPq) and Programa de Apoio ao Desenvolvimento Científico (PADCFCF-UNESP) for financial support. This project was supported by São Paulo Research Foundation (FAPESP) by grant number: 13/03746-3; and 15/05394-2.

\section{Author contributions}

BFS designed the study, analyzed the data, and drafted the manuscript. AMS and CFR collaborated the in vivo antiinflammatory experiments and contributed to the drafting of the manuscript. MPDG and MC coordinated the research and drafted the manuscript. All authors contributed toward data analysis, drafting and revising the paper and agree to be accountable for all aspects of the work. 


\section{Disclosure}

The authors report no conflicts of interest in this work.

\section{References}

1. Eastoe J. Surfactant aggregation and adsorption at interfaces. In: Cosgrove T, editor. Colloid Science: Principles, Methods and Applications. 2nd ed. Southern Gate: Wiley; 2010:61-90.

2. Pershan PS. Lyotropic liquid crystals. Phys Today. 1982;35(5):34-39.

3. Tiddy GJT. Surfactant-water liquid crystal phases. Phys Rep. 1980;57(1): $1-46$.

4. Kim DH, Jahn A, Cho SJ, Kim JS, Ki MH, Kim DD. Lyotropic liquid crystal systems in drug delivery: a review. J Pharma Investig. 2015;45(1): $1-11$.

5. Guo C, Wang J, Cao F, Lee RJ, Zhai G. Lyotropic liquid crystal systems in drug delivery. Drug Discov Today. 2010;15(23-24):1032-1040.

6. Boyd BJ, Whittaker DV, Khoo S-M, Davey G. Lyotropic liquid crystalline phases formed from glycerate surfactants as sustained release drug delivery systems. Int J Pharma. 2006;309(1-2):218-226.

7. Nielsen LS, Schubert L, Hansen J. Bioadhesive drug delivery systems: I. characterisation of mucoadhesive properties of systems based on glyceryl mono-oleate and glyceryl monolinoleate. Eur J Pharma Sci. 1998;6(3):231-239.

8. Martiel I, Baumann N, Vallooran JJ, Bergfreund J, Sagalowicz L, Mezzenga R. Oil and drug control the release rate from lyotropic liquid crystals. J Control Release. 2015;204:78-84.

9. Sagalowicz L, Leser ME, Watzke HJ, Michel M. Monoglyceride self-assembly structures as delivery vehicles. Trends Food Sci Tech. 2006;17(5):204-214.

10. Phelps J, Bentley MVLB, Lopes LB. In situ gelling hexagonal phases for sustained release of an anti-addiction drug. Colloids Surf B Biointerfaces. 2011;87(2):391-398.

11. Nesseem DI. Formulation and evaluation of itraconazole via liquid crystal for topical delivery system. J Pharma Biomed Anal. 2001;26(3): 387-399.

12. Yamada K, Yamashita J, Todo H, et al. Preparation and evaluation of liquid-crystal formulations with skin-permeation-enhancing abilities for entrapped drugs. J Oleo Sci. 2011;60(1):31-40.

13. Carvalho FC, e Silva HR, da Luz GM, et al. Rheological, mechanical and adhesive properties of surfactant-containing systems designed as a potential platform for topical drug delivery. J Biomed Nanotech. 2012; 8(2):280-289.

14. Müller-Goymann CC. Physicochemical characterization of colloidal drug delivery systems such as reverse micelles, vesicles, liquid crystals and nanoparticles for topical administration. Eur J Pharma Biopharma. 2004;58(2):343-356.

15. Borgheti-Cardoso LN, Depieri LV, Diniz H, et al. Self-assembling gelling formulation based on a crystalline-phase liquid as a non-viral vector for siRNA delivery. Eur J Pharma Sci. 2014;58:72-82.

16. Borgheti-Cardoso LN, Depieri LV, Kooijmans SAA, et al. An in situ gelling liquid crystalline system based on monoglycerides and polyethylenimine for local delivery of siRNAs. Eur J Pharma Sci. 2015; 74:103-117.

17. Jurenka JS. Anti-inflammatory properties of curcumin, a major constituent of Curcuma longa: a review of preclinical and clinical research. Altern Med Rev. 2009;14(2):141-153.

18. Chainani-Wu N. Safety and anti-inflammatory activity of curcumin: a component of tumeric (Curcuma longa). J Altern Complement Med. 2003;9(1):161-168.

19. Thangapazham RL, Sharad S, Maheshwari RK. Skin regenerative potentials of curcumin. Biofactors. 2013;39(1):141-149.

20. Nguyen TA, Friedman AJ. Curcumin: a novel treatment for skin-related disorders. J Drugs Dermatol. 2013;12(10):1131-1137.

21. Akbik D, Ghadiri M, Chrzanowski W, Rohanizadeh R. Curcumin as a wound healing agent. Life Sci. 2014;116(1):1-7.
22. Carrion-Gutierrez M, Ramirez-Bosca A, Navarro-Lopez V, et al. Effects of Curcuma extract and visible light on adults with plaque psoriasis. Eur J Dermatol. 2015;25(3):240-246.

23. Sun J, Zhao Y, Hu J. Curcumin inhibits imiquimod-induced psoriasislike inflammation by inhibiting IL-1beta and IL-6 production in mice. PLoS One. 2013;8(6):e67078.

24. Ryan JL, Heckler CE, Ling M, et al. Curcumin for radiation dermatitis: a randomized, double-blind, placebo-controlled clinical trial of thirty breast cancer patients. Radiat Res. 2013;180(1):34-43.

25. Hata M, Sasaki E, Ota M, et al. Allergic contact dermatitis from curcumin (turmeric). Contact Dermatitis. 1997;36(2):107-108.

26. Phillips JM, Clark C, Herman-Ferdinandez L, et al. Curcumin inhibits skin squamous cell carcinoma tumor growth in vivo. Otolaryngol Head Neck Surg. 2011;145(1):58-63.

27. Abusnina A, Keravis T, Yougbaré I, Bronner C, Lugnier C. Antiproliferative effect of curcumin on melanoma cells is mediated by PDE1A inhibition that regulates the epigenetic integrator UHRF1. Molecul Nutrit Food Res. 2011;55(11):1677-1689.

28. Sonavane K, Phillips J, Ekshyyan O, et al. Topical curcumin-based cream is equivalent to dietary curcumin in a skin cancer model. J Skin Cancer. 2012;2012:147863.

29. Anand P, Kunnumakkara AB, Newman RA, Aggarwal BB. Bioavailability of curcumin: problems and promises. Molecul Pharma. 2007; 4(6):807-818.

30. Chen Y, Wu Q, Zhang Z, Yuan L, Liu X, Zhou L. Preparation of curcumin-loaded liposomes and evaluation of their skin permeation and pharmacodynamics. Molecules. 2012;17(5):5972-5987.

31. Mangalathillam S, Rejinold NS, Nair A, Lakshmanan V-K, Nair SV, Jayakumar R. Curcumin loaded chitin nanogels for skin cancer treatment via the transdermal route. Nanoscale. 2012;4(1): 239-250.

32. Mathiowitz E, Chickering DE, Lehr CM. Bioadhesive Drug Delivery Systems: Fundamentals, Novel Approaches, and Development. New York, NY: CRC Press; 1999.

33. Chen Y, Ma P, Gui S. Cubic and hexagonal liquid crystals as drug delivery systems. Biomed Res Int. 2014;2014:815981.

34. Silva H, da Luz GM, Satake CY, et al. Surfactant-based transdermal system for fluconazole skin delivery. J Nanomed Nanotech. 2014; $5(5): 231$.

35. Urban MCC, Landgraf DS, Oyafuso MH, et al. Development and in vitro skin delivery of dexamethasone acetate-loaded surfactant-based systems. J Nanopharma Drug Deliv. 2013;1(3):323-334.

36. Oliveira MB, Prado AHd, Bernegossi J, et al. Topical application of retinyl palmitate-loaded nanotechnology-based drug delivery systems for the treatment of skin aging. Biomed Res Int. 2014;2014: 632570 .

37. Gonzalez ML, Corrêa MA, Chorilli M. Skin delivery of kojic acidloaded nanotechnology-based drug delivery systems for the treatment of skin aging. Biomed Res Int. 2013;2013:271276.

38. Carvalho FC, Sarmento VHV, Chiavacci LA, Barbi MS, Gremião MPD. Development and in vitro evaluation of surfactant systems for controlled release of zidovudine. J Pharma Sci. 2010;99(5): 2367-2374.

39. Carvalho FC, Campos ML, Peccinini RG, Gremião MPD. Nasal administration of liquid crystal precursor mucoadhesive vehicle as an alternative antiretroviral therapy. Eur J Pharma Biopharma. 2013;84(1): 219-227.

40. Calixto G, Yoshii AC, Rocha e Silva H, Stringhetti Ferreira Cury B, Chorilli M. Polyacrylic acid polymers hydrogels intended to topical drug delivery: preparation and characterization. Pharma Develop Tech. 2015;20(4):490-496.

41. Cintra GA, Pinto LA, Calixto GM, et al. Bioadhesive surfactant systems for methotrexate skin delivery. Molecules. 2016;21(2):pii: E231.

42. Dick IP, Scott RC. Pig ear skin as an in-vitro model for human skin permeability. J Phar Pharmacol. 1992;44(8):640-645. 
43. Carvalho FC, Campos ML, Peccinini RG, Gremiao MP. Nasal administration of liquid crystal precursor mucoadhesive vehicle as an alternative antiretroviral therapy. Eur J Pharma Biopharma. 2013;84(1): 219-227.

44. Malmsten M. Surfactants and Polymers in Drug Delivery. New York, NY: Infoma Healthcare; 2002.

45. Malmsten M. Phase transformations in self-assembly systems for drug delivery applications. J Dispers Sci Tech. 2007;28(1):63-72.

46. Israelachvili JN, Mitchell DJ, Ninham BW. Theory of self-assembly of hydrocarbon amphiphiles into micelles and bilayers. J Chem Soc, Faraday Trans 2. 1976;72(0):1525-1568.

47. Israelachvili JN. Intermolecular and Surfaces Forces. 2nd ed. New York, NY: Academic; 1991.

48. Li Q. Nanoscience with Liquid Crystals: From Self-Organized Nanostructures to Applications. New York, NY: Springer; 2014.

49. Mezzenga R. Physics of self-assembly of lyotropic liquid crystals. In: Garti N, Somasundaran P, Mezzenga R, editors. Self-assembled Supramolecular Architectures: Lyotropic Liquid Crystals. Hoboken: Wiley; 2012:1-20

50. Hyde ST. Identification of lyotropic liquid crystalline mesophases. In: Holmberg K, editor. Handbook of Applied Surface and Colloid Chemistry. Vol 2. New York, NY: Wiley; 2002:299-332.

51. Garti N, Libster D, Aserin A. Lipid polymorphism in lyotropic liquid crystals for triggered release of bioactives. Food Funct. 2012;3(7): 700-713.

52. Kaasgaard T, Drummond CJ. Ordered 2-D and 3-D nanostructured amphiphile self-assembly materials stable in excess solvent. Phys Chem Chem Phys. 2006;8(43):4957-4975.

53. Patel R, Patel TN. Liquid crystals and their application in the field of drug delivery. In: Fanun M, editor. Colloids in Drug Delivery. Boca Raton: CRC Press; 2010:311-336.

54. Yaghmur A, de Campo L, Salentinig S, Sagalowicz L, Leser ME, Glatter O. Oil-loaded monolinolein-based particles with confined inverse discontinuous cubic structure (Fd3m). Langmuir. 2006 22(2):517-521.

55. Soni SS, Brotons G, Bellour M, Narayanan T, Gibaud A. Quantitative SAXS analysis of the $\mathrm{p} 123$ /water/ethanol ternary phase diagram. J Phys Chem B. 2006;110(31):15157-15165.

56. Gunasekaran S, Ak MM. Dynamic oscillatory shear testing of foods selected applications. Trends Food Sci Tech. 2000;11(3):115-127.

57. Mezger TG. The Rheology Handbook: For Users of Rotational and Oscillatory Rheometers. 2nd ed. Hannover: Vincentz Network; 2014.

58. Carvalho FC, Calixto G, Hatakeyama IN, Luz GM, Gremião MPD, Chorilli M. Rheological, mechanical, and bioadhesive behavior of hydrogels to optimize skin delivery systems. Drug Develop Indus Phar. 2012;39(11):1750-1757.

59. Chorilli M, Rigon RB, Calixto G, et al. Rheological characterization and safety evaluation of non-ionic lamellar liquid crystalline systems containing retinyl palmitate. J Biomed Nanotechnol. 2016;12(2): 394-403.

60. Breene WM. Application of texture profile analysis to instrumental food texture evaluation. J Texture Stud. 1975;6(1):53-82.

61. Tamburic S, Craig DQM. A comparison of different in vitro methods for measuring mucoadhesive performance. Eur J Pharma and Biopharma 1997;44(2):159-167.

62. Andrews GP, Donnelly L, Jones DS, et al. Characterization of the rheological, mucoadhesive, and drug release properties of highly structured gel platforms for intravaginal drug delivery. Biomacromolecules. 2009;10(9):2427-2435.

63. Friedman HH, Whitney JE, Szczesniak AS. The texturometer - a new instrument for objective texture measurement. J Food Sci. 1963;28(4): 390-396.

64. Jones DS, Woolfson AD, Djokic J. Texture profile analysis of bioadhesive polymeric semisolids: mechanical characterization and investigation of interactions between formulation components. J Appl Polym Sci. 1996;61(12):2229-2234
65. Oyafuso MH, Carvalho FC, Chiavacci LA, Gremião MPD, Chorilli M. Design and characterization of silicone and surfactant based systems for topical drug delivery. J Nanosci Nanotechnol. 2015;15(1):817-826.

66. Jones DS, Irwin CR, David Woolfson A, Djokic J, Adams V. Physicochemical characterization and preliminary in vivo efficacy of bioadhesive, semisolid formulations containing flurbiprofen for the treatment of gingivitis. J Pharma Sci. 1999;88(6):592-598.

67. Estanqueiro M, Amaral MH, Sousa Lobo JM. Comparison between sensory and instrumental characterization of topical formulations: impact of thickening agents. Int J Cosmet Sci. 2016;38(4):389-398.

68. Jones DS, Woolfson AD, Brown AF. Textural, viscoelastic and mucoadhesive properties of pharmaceutical gels composed of cellulose polymers. Int J Pharma. 1997;151(2):223-233.

69. Carvalho FC, Bruschi ML, Evangelista RC, Gremião MPD. Mucoadhesive drug delivery systems. Braz J Pharma Sci. 2010;46:1-17.

70. Smart JD. The basics and underlying mechanisms of mucoadhesion. Adv Drug Deliv Rev. 2005;57(11):1556-1568.

71. Gandhi RB, Robinson JR. Oral cavity as a site for bioadhesive drug delivery. Adv Drug Deliv Rev. 1994;13(1-2):43-74.

72. Ugwoke MI, Agu RU, Verbeke N, Kinget R. Nasal mucoadhesive drug delivery: background, applications, trends and future perspectives. $A d v$ Drug Deliv Rev. 2005;57(11):1640-1665.

73. Robinson JR, Mlynek GM. Bioadhesive and phase-change polymers for ocular drug delivery. Adv Drug Deliv Rev. 1995;16(1):45-50.

74. Mansuri S, Kesharwani P, Jain K, Tekade RK, Jain NK. Mucoadhesion: a promising approach in drug delivery system. React Funct Polym. 2016; 100:151-172.

75. Agarwal S, Aggarwal S. Mucoadhesive polymeric platform for drug delivery. A comprehensive review. Curr Drug Deliv. 2015;12(2): $139-156$.

76. Mikos AG, Peppas NA. Systems for controlled release of drugs. V. Bioadhesive systems. Sci Tech Pra Pharma. 1986;2(19):705-716.

77. Peppas NA, Sahlin JJ. Hydrogels as mucoadhesive and bioadhesive materials: a review. Biomaterials. 1996;17(16):1553-1561.

78. Peppas NA, Huang Y. Nanoscale technology of mucoadhesive interactions. Adv Drug Deliv Rev. 2004;56(11):1675-1687.

79. Anurova MN, Bakhrushina EO, Demina NB. Review of contemporary gel-forming agents in the technology of dosage forms. Pharma Chem J. 2015;49(9):627-634.

80. Casalini T, Perale G. Types of bioresorbable polymers for medical applications. In: Durability and Reliability of Medical Polymers. 2012:3-29.

81. Patel HR, Patel RP, Patel MM. Poloxamers: a pharmaceutical excipients with therapeutic behaviors. Int J Pharmtech Res. 2009;1(2):299-303.

82. De Souza Ferreira SB, Moço TD, Borghi-Pangoni FB, Junqueira MV, Bruschi ML. Rheological, mucoadhesive and textural properties of thermoresponsive polymer blends for biomedical applications. J Mech Behav Biomed Mater. 2015;55:164-178.

83. Borghi-Pangoni FB, Junqueira MV, De Souza Ferreira SB, et al. Screening and in vitro evaluation of mucoadhesive thermoresponsive system containing methylene blue for local photodynamic therapy of colorectal cancer. Pharma Res. 2016;33(3):776-791.

84. Junqueira MV, Borghi-Pangoni FB, Ferreira SBS, Rabello BR, Hioka N, Bruschi ML. Functional polymeric systems as delivery vehicles for methylene blue in photodynamic therapy. Langmuir. 2016;32(1): 19-27.

85. Jones DS, Bruschi ML, de Freitas O, Gremião MPD, Lara EHG, Andrews GP. Rheological, mechanical and mucoadhesive properties of thermoresponsive, bioadhesive binary mixtures composed of poloxamer 407 and carbopol 974P designed as platforms for implantable drug delivery systems for use in the oral cavity. Int J Pharma. 2009; 372(1-2):49-58.

86. Du JD, Liu Q, Salentinig S, Nguyen TH, Boyd BJ. A novel approach to enhance the mucoadhesion of lipid drug nanocarriers for improved drug delivery to the buccal mucosa. Int J Pharma. 2014;471(1-2): $358-365$. 
87. Lee J, Young SA, Kellaway IW. Water quantitatively induces the mucoadhesion of liquid crystalline phases of glyceryl monooleate. J Pharm Pharmacol. 2001;53(5):629-636.

88. Nielsen LS, Schubert L, Hansen J. Bioadhesive drug delivery systems. I. Characterisation of mucoadhesive properties of systems based on glyceryl mono-oleate and glyceryl monolinoleate. Eur J Pharma Sci. 1998;6(3):231-239.

89. Ramos MAS, Calixto G, de Toledo LG, et al. Liquid crystal precursor mucoadhesive system as a strategy to improve the prophylactic action of Syngonanthus nitens (Bong.) Ruhland against infection by Candida krusei. Int J Nanomed. 2015;10:7455-7466.
90. Souza C, Watanabe E, Borgheti-Cardoso LN, De Fantini MCA, Lara MG. Mucoadhesive system formed by liquid crystals for buccal administration of poly(hexamethylene biguanide) hydrochloride. J Pharma Sci. 2014;103(12):3914-3923.

91. Ruela ALM, Carvalho FC, Pereira GR. Exploring the phase behavior of monoolein/oleic acid/water systems for enhanced donezepil administration for alzheimer disease treatment. J Pharma Sci. 2016;105(1): 71-77.

92. Bernegossi J, Calixto G, Sanches P, et al. Peptide KSL-W-Loaded mucoadhesive liquid crystalline vehicle as an alternative treatment for multispecies oral biofilm. Molecules. 2016;21(1):37.

\section{Publish your work in this journal}

The International Journal of Nanomedicine is an international, peerreviewed journal focusing on the application of nanotechnology in diagnostics, therapeutics, and drug delivery systems throughout the biomedical field. This journal is indexed on PubMed Central, MedLine, CAS, SciSearch $®$, Current Contents ${ }^{\circledR} /$ Clinical Medicine,
Journal Citation Reports/Science Edition, EMBase, Scopus and the Elsevier Bibliographic databases. The manuscript management system is completely online and includes a very quick and fair peer-review system, which is all easy to use. Visit http://www.dovepress.com/ testimonials.php to read real quotes from published authors. 